\title{
The Influence of Cosolvent Polarity on the Flow Properties of Hydroalcoholic Gels. Empirical Models
}

\author{
María Magdalena Jiménez, María José Fresno, ${ }^{*}$ and Alain Ramírez \\ Department of Pharmacy and Pharmaceutical Technology, Faculty of Pharmacy, Alcalá University; Campus Universitario \\ E-28871 Alcalá de Henares, Madrid, Spain. Received February 28, 2005; accepted May 30, 2005
}

\begin{abstract}
The aim of this study was to investigate the influence of cosolvent polarity on the rheological flow properties of aqueous and hydroalcoholic gels obtained from the Carbopol ${ }^{\circledR}$ Ultrez $^{\mathrm{TM}} 10$ base and used for topical applications. Specifically, we have examined the effect of $\mathrm{pH}$ (range 4.0-7.0) on the consistency and flow properties of dispersed systems in water and mixtures- $15: 85 \% \mathrm{v} / \mathrm{v}$ of methanol : water, ethanol : water, $n$-propanol : water and $n$-butanol : water-at a constant polymer concentration of $0.3 \% \mathrm{w} / \mathrm{w}$. The gels, which had decreasing polarity values in the jellifying medium, showed qualitatively similar flow behavior, characteristic of pseudoplastic systems, and all of the flow curves were adjusted to the Ostwald model. Sigmoidal dose response functions were calculated to describe the flow and consistency indexes as a function of $\mathrm{pH}$. As a result, the influence of alcohol polarity on the polymer network has been assessed meaningfully using the empirical parameters obtained: maximum consistency index value $\left(k_{\max }\right)$, $\mathrm{pH}$ value required for $50 \%$ development of polymer network $\left(\mathrm{pH}_{50}\right)$, and asymptotic flow index value $\left(n_{\min }\right)$ for the fully structured gels.
\end{abstract}

Key words Carbopol $^{\circledR}$ Ultrez $^{\mathrm{TM}} 10(0.3 \% \mathrm{w} / \mathrm{w})$; hydroalcoholic gels; cosolvent polarity; flow behavior; empirical models

Cosolvents have been used widely as vehicles in the formulation of semisolid bases, generally with a significant influence on their rheological properties. When organic amines are employed as neutralizing agents of carbopols, it is possible to gel many semipolar liquids and mixtures of these liquids with water. Compatibility of the polymer and non-polar solvents depends on the formation of ion pairs with the amine. ${ }^{1,2}$

Although alcohols are useful for increasing the solubility of non-polar drugs, their use as cosolvents with hydrophilic polymers is often limited by potential physical incompatibilities among them. The rheological behavior of polymer solutions is determined not only by the polymer-polymer conformation and entanglement, but also by the concentration and polymer-solvent effects. Consequently, the compatibility of the gelling agent and non-aqueous solvent determines the alcohol content to be used in designing and developing technologically adequate hydroalcoholic gelled bases. ${ }^{3,4)}$

Carbopols are synthetic cross-linked acrylic acid polymers used widely in the pharmaceutical and cosmetic industries. ${ }^{5}$ In recent years hydrogels obtained from these polymers have increasingly been employed as vehicles for pharmaceutical preparations. ${ }^{6-9)}$ This kind of polymers are known for producing gels that depend on the $\mathrm{pH}$ of the medium. Neutralization causes dissociation of the carboxylic groups and expansion, through electrostatic repulsion, of the polymer chains, which form a three-dimensional network to gel the medium. For this reason, the $\mathrm{pH}$ of the medium is one of the most important parameters in their formulation and future characterization. The rheological and pharmacotechnical properties of these systems are determined by the extent of structural development in the network. ${ }^{10-13)}$

Cross-linked acrylic acid polymers are already well-known for their unique rheological properties, such as the ability to impart vertical cling and a "no-drip" rheology. These unique properties can enhance the performance characteristics of applications. This kind of polymers also provides a shear thinning rheology and the ability to recover viscosity immediately after shearing has ceased, thus enabling the gel to be applied or squeezed out of a bottle easily. In addition, they are odour free and have a high buffering capability due to the large number of carboxylic acid groups on the polymer, which helps to maintain a stable $\mathrm{pH}$ in the finished product and during its use.

Carbopol $^{\mathbb{B}}$ Ultrez $^{\mathrm{TM}} 10$, a carbomer polymer, is considered to be a universal polymer capable of replacing two or more carbomer polymer grades currently in use to formulate lotions, creams and gels. ${ }^{14)}$ It is a special case of nonlinear, branched polymer chains which interconnect to form a threedimensional network structure. In dispersions consisting of network polymers the dominant structural feature is (water) swollen microgels. Polymer microgel particles increase the viscosity of a solution essentially through two mechanisms: high crosslink density and high microgel rigidity. It is interesting to note here that both high crosslink density and high microgel rigidity mechanisms, as present in Carbopol ${ }^{\circledR}$ Ultrez ${ }^{\mathrm{TM}} 10$, yield the most efficient carbomer polymers for viscosity building.

An interesting crossover phenomenon occurs because of the dual nature of viscosity building through carbomer polymers. Low swelling carbomer polymers (e.g. Carbopol ${ }^{\mathbb{B}}$ Ultrez $\left.^{\mathrm{TM}} 10, \mathrm{C}-934\right)$ show less viscosity at lower polymer concentrations; however, the situation is reversed at higher polymer concentrations. This illustrates the unique rigid particle nature of Carbopol ${ }^{\mathbb{B}}$ Ultrez $^{\mathrm{TM}} 10$ resin compared to the traditional C-940 and C-934 resins. In fact, the most efficient data reported in this paper show that the Carbopol ${ }^{\circledR}$ Ultrez $^{\mathrm{TM}} 10$ carbomer polymer is capable of replacing either of these resins in certain dermopharmaceutical product formulations. The rheological parameters of Carbopol ${ }^{\circledR}$ Ultrez $^{\mathrm{TM}} 10$ resin compared to the traditional C-941, C-940 and C-934 resins and quantitative values for each parameter have been published by BFGoodrich Company. ${ }^{15)}$

Increased interest in these polymers for the elaboration of cosmetic and pharmaceutical formulations, and particularly the advantages and new nature of Carbopol ${ }^{\circledR}$ Ultrez $^{\mathrm{TM}} 10$, justify an exhaustive rheological study of the gelled topical forms of the polymer as a function of neutralization and the 
content and polarity of the cosolvent. Specifically, our objective is to obtain empirical mathematical models to correlate the rheological parameters and the variables in question in order to establish a priori the hydrogel composition needed for specific applications in which rheological properties are determining factors.

Gelled water-soluble bases can be designed and formulated to optimize topical release of non-polar drugs. Specifically, rheological properties have an evident, proven, fundamental influence on release parameters, meaning that an exhaustive rheological and structural characterization can facilitate use of empirical parameters and models to optimize topical release from dermatological and cosmetic formulations. $^{16-21)}$

The specific objective of this work is to study the influence of polarity on the empirical variables $-k_{\max }, \mathrm{pH}_{50}$ and $n_{\min }-$ by evaluating the dependence of the jellifying process on $\mathrm{pH}$ $(4.0-7.0)$ in mixtures of $15: 85 \% \mathrm{v} / \mathrm{v}$ methanol: water, ethanol: water, $n$-propanol: water and $n$-butanol: water for a specific polymer concentration of $0.3 \% \mathrm{w} / \mathrm{w}$. Empirical rheological models before obtained ${ }^{2,22)}$ are also applied to evaluate the influence of cosolvent polarity on the rheological flow properties of gels. It is particularly essential to evaluate their potential use as dermatological and/or cosmetic bases when alcohol mixtures are employed to increase solubility.

\section{Experimental}

Materials The materials used to produce the gelled medium were double distilled deionized Milli- ${ }^{\circledR}$ water, methanol, ethanol, $n$-propanol and $n$ butanol, all of pure quality for analysis (Scharlau, Barcelona, Spain), and Carbopol $^{\circledR}$ Ultrez $^{\mathrm{TM}} 10$ supplied by BF Goodrich Chemical Co. (Cleveland, Ohio, U.S.A.). The $50 \%$ triethanolamine solution was obtained by dissolution of triethanolamine PA (E. Merck, Darmstadt, Germany) in Milli- $Q^{\mathbb{R}}$ water.

Procedure Exact quantities of $1.50 \mathrm{~g}$ of $\mathrm{Carbopol}^{\circledR} \mathrm{Ultrez}^{\mathrm{TM}} 10$ were weighed and added to $500 \mathrm{ml}$ of distilled deionized water or to mixtures of ethanol: water, methanol:water, $n$-propanol: water and $n$-butanol: water prepared in proportions of $15: 85 \mathrm{v} / \mathrm{v}$, to obtain a final polymer concentration of $0.3 \% \mathrm{w} / \mathrm{w}$. The mixtures were agitated at $2000 \mathrm{rpm}$ for $10 \mathrm{~min}$ with a Silverson L4R agitator, equipped with an axial flow head and disintegrating head.

The dispersions were set aside for one hour in order to eliminate any air, and were then neutralized with a $50 \%$ triethanolamine solution to obtain the required $\mathrm{pH}(4.0 ; 4.5 ; 5.0 ; 5.5 ; 6.0 ; 6.5 ; 7.0)$. Homogenization during neutralization was carried out at a continuous flow at $1000 \mathrm{rpm}$ to prevent air from entering the gelled mass. The $\mathrm{pH}$ measurements were taken in situ with a digital Crison 2001 micro $\mathrm{pH}$-meter, previously calibrated and equipped with an electrode for emulsions. The samples were allowed to equilibrate for $72 \mathrm{~h}$ prior to evaluation of their rheological properties. ${ }^{23,24)}$

Determination of the Flow Curves Viscosity was determined using a cone and plate Brookfield Digital Viscometer DV-III, equipped with a recirculating Brookfield TC 200 bath to control the sample-container temperature. Rheograms were obtained for 11 decreasing shear rates $\left(D, \mathrm{~s}^{-1}\right)$ in the range $2-200 \mathrm{~s}^{-1}$ using the $\mathrm{CP}-52$ cone. The reproducibility of the data was assessed by determining the rheograms at $20.0 \pm 0.1{ }^{\circ} \mathrm{C}$ for $0.5 \mathrm{ml}$ of each sample $(n=3) .{ }^{25)}$ The temperature of each sample on the rheometer plate was allowed to stabilize for five minutes before the viscosity measurement was taken. The rheograms obtained were analyzed and adjusted to the Ostwald model using Rheocalc for Windows, version 1.01.

Determination of Mathematical Models The results were analyzed using the statistical software GraphPad Prism, version 1.0, and Microsoft ${ }^{\mathbb{B}}$ Excel 2000 in order to obtain linear and non-linear mathematical models that would correlate the rheological parameters of the dispersed systems studied and the variables of $\mathrm{pH}$ and cosolvent polarity. ${ }^{26)}$

The specific objective were to obtain empirical mathematical models that correlate the rheological parameters and the variables studied to establish $a$ priori the gel composition needed for a specific application in which rheological properties are a determining factor; these models could be applied to the rational design of formulations for topical application and release of non-polar drugs. ${ }^{27-29)}$

\section{Results and Discussion}

The Carbopol ${ }^{\circledR}$ Ultrez $^{\mathrm{TM}} 10$ hydroalcoholic gels, which had decreasing polarity values in the jellifying medium (methanol $>$ ethanol $>n$-propanol $>n$-butanol), showed a qualitatively similar flow behaviour, characteristic of pseudoplastic systems. All of the rheograms obtained were adjusted to the Ostwald model $\left(\tau=k \cdot D^{n}\right)$, with correlation coefficients over 0.9900 . This finding shows the constant nature of the flow mechanism in the different alcohol mixtures studied since the power law is able to reproduce adequately the rheological behaviour of these dispersed systems. In all cases, the values of $k$ and $n$ were used to objectively establish the modifications produced by the degree of U10 neutralization upon the rheological behaviour of the gel.

Table 1 shows the values for the consistency index $\left(\bar{k} \pm\right.$ S.D., $\left.\mathrm{mPa} \cdot \mathrm{s}^{n}\right)$ and flow index $(\bar{n} \pm$ S.D., adimensional $)$ of the formulations as a function of the cosolvent concentration, the total cosolvent solubility parameter $\left[\delta_{\mathrm{T}} ;\left(\mathrm{cal} / \mathrm{cm}^{3}\right)^{1 / 2}\right]$ and $\mathrm{pH}^{30)}$

Consistency Indexes The calculated power law consistency indexes $(k)$ were well correlated to $\mathrm{pH}$. The relationship between the mean consistency index of the hydroalco-

Table 1. Values of the Consistency Indexes $\left(\bar{k}, \mathrm{mPa} \cdot \mathrm{s}^{n}\right)$ and the Flow Indexes ( $n$, Adimensional) of the Carbopol ${ }^{\mathbb{B}}$ Ultrez $^{\mathrm{TM}} 10$ Gels as a Function of Cosolvent Concentration, Total Cosolvent Solubility Parameter $\left[\delta_{\mathrm{T}}\right.$; $\left.\left(\mathrm{cal} / \mathrm{cm}^{3}\right)^{1 / 2}\right]$ and $\mathrm{pH}$

\begin{tabular}{|c|c|c|c|c|}
\hline $\begin{array}{l}\text { Cosolvent } \\
(\%, \mathrm{v} / \mathrm{v})\end{array}$ & $\underset{\left(\mathrm{cal} / \mathrm{cm}^{3}\right)^{1 / 2}}{\delta_{\mathrm{T}}}$ & $\mathrm{pH}$ & $\bar{k}\left(\mathrm{mPa} \cdot \mathrm{s}^{n}\right) \pm \mathrm{S} . \mathrm{D} .^{a}{ }^{a}$ & $\bar{n} \pm \mathrm{S} . \mathrm{D}$. \\
\hline \multirow[t]{7}{*}{$100 \%$ Water } & 23.4 & 4.0 & $54886 \pm 1913.2$ & $0.22 \pm 0.0006$ \\
\hline & & 4.5 & $59608 \pm 4787.2$ & $0.21 \pm 0.0007$ \\
\hline & & 5.0 & $61976 \pm 4443.9$ & $0.21 \pm 0.0012$ \\
\hline & & 5.5 & $62378 \pm 1016.6$ & $0.21 \pm 0.0011$ \\
\hline & & 6.0 & $62927 \pm 696.0$ & $0.21 \pm 0.0011$ \\
\hline & & 6.5 & $63039 \pm 3360.9$ & $0.21 \pm 0.0013$ \\
\hline & & 7.0 & $63412 \pm 3791.1$ & $0.21 \pm 0.0013$ \\
\hline \multirow[t]{7}{*}{ 15\% Methanol } & 14.5 & 4.0 & $50011 \pm 414.7$ & $0.23 \pm 0.0034$ \\
\hline & & 4.5 & $55336 \pm 622.9$ & $0.22 \pm 0.0025$ \\
\hline & & 5.0 & $58455 \pm 970.4$ & $0.22 \pm 0.0021$ \\
\hline & & 5.5 & $60040 \pm 587.7$ & $0.21 \pm 0.0021$ \\
\hline & & 6.0 & $60569 \pm 836.1$ & $0.21 \pm 0.0023$ \\
\hline & & 6.5 & $60611 \pm 704.8$ & $0.21 \pm 0.0020$ \\
\hline & & 7.0 & $60652 \pm 880.7$ & $0.21 \pm 0.0022$ \\
\hline \multirow[t]{7}{*}{$15 \%$ Ethanol } & 13.0 & 4.0 & $45020 \pm 246.07$ & $0.25 \pm 0.0031$ \\
\hline & & 4.5 & $50745 \pm 417.58$ & $0.24 \pm 0.0023$ \\
\hline & & 5.0 & $54447 \pm 459.15$ & $0.23 \pm 0.0020$ \\
\hline & & 5.5 & $56179 \pm 369.97$ & $0.22 \pm 0.0022$ \\
\hline & & 6.0 & $56432 \pm 522.88$ & $0.23 \pm 0.0027$ \\
\hline & & 6.5 & $56617 \pm 527.77$ & $0.22 \pm 0.0022$ \\
\hline & & 7.0 & $56941 \pm 574.37$ & $0.22 \pm 0.0024$ \\
\hline \multirow[t]{7}{*}{$15 \% n$-Propanol } & 12.0 & 4.0 & $24553 \pm 209.74$ & $0.30 \pm 0.0057$ \\
\hline & & 4.5 & $36410 \pm 395.41$ & $0.29 \pm 0.0059$ \\
\hline & & 5.0 & $43293 \pm 903.24$ & $0.27 \pm 0.0057$ \\
\hline & & 5.5 & $48658 \pm 331.18$ & $0.27 \pm 0.0047$ \\
\hline & & 6.0 & $50704 \pm 190.29$ & $0.25 \pm 0.0047$ \\
\hline & & 6.5 & $51293 \pm 402.73$ & $0.25 \pm 0.0046$ \\
\hline & & 7.0 & $51558 \pm 433.44$ & $0.24 \pm 0.0034$ \\
\hline \multirow[t]{6}{*}{$15 \% n$-Butanol } & 11.3 & 4.5 & $9994 \pm 286.3$ & $0.38 \pm 0.0053$ \\
\hline & & 5.0 & $25951 \pm 728.1$ & $0.36 \pm 0.0056$ \\
\hline & & 5.5 & $35462 \pm 818.8$ & $0.34 \pm 0.0048$ \\
\hline & & 6.0 & $40754 \pm 1543.2$ & $0.31 \pm 0.0049$ \\
\hline & & 6.5 & $45359 \pm 975.5$ & $0.30 \pm 0.0038$ \\
\hline & & 7.0 & $47845 \pm 905.1$ & $0.29 \pm 0.0045$ \\
\hline
\end{tabular}

a) Standard deviation. 
holic gels and $\mathrm{pH}$ for the jellifying medium compositions is represented in graph form in Fig. 1.

It can be seen that dependence of the $k$ values and $\mathrm{pH}$ is qualitatively similar to the $k-\mathrm{pH}$ profile obtained previously for mixtures of ethanol: water gelled with Carbopol ${ }^{\circledR}$ Ultrez ${ }^{\mathrm{TM}} 10^{2,22)}$ Considering the importance of cosolvents in developing dermatological bases, we extended the application of the model to a wider series of decreasing polarity alcohols.

The $k$-values show an initial pronounced increase with increasing $\mathrm{pH}$ (in the range $4.0-5.5$ ) and a further stabilisation or reduced increment for $\mathrm{pH}$ values between 5.5 and 7.0 (Fig. 1). This behaviour indicates an initial more noticeable progressive development of polymer network with increasing $\mathrm{pH}$ which attenuates for $\mathrm{pH}$ increments in the range 5.57.0. This behaviour can be explained by the molecular structure of the gel. In fact, the polymer chains are initially (i.e. at $\mathrm{pH}$ 4) coiled into a spiral form, thus affording a relatively low viscosity. As neutralization progresses, the carboxyl groups of the acrylic acid become ionized, leading to an increasing repulsion of negative charges that causes the molecular structure to unwind, thus inducing a gradual rise in viscosity. The mean $k$ values for the alcohol: water mixtures studied were correlated with $\mathrm{pH}$ according to a sigmoidal dose response function (Eq. 1) of the type:

$$
k=\frac{k_{\max }}{1+10^{\left(\mathrm{pH}_{50}-\mathrm{pH}\right)}}
$$

According to this expression, consistency of hydroalcoholic gels increased with $\mathrm{pH}$ and tended to a maximum value $\left(k_{\max }\right)$ at neutral $\mathrm{pH}$, which was in agreement with the general behaviour of acrylic acid polymers upon neutralisation. Furthermore, the variable $\mathrm{pH}_{50}$ introduced in Eq. 1, determined

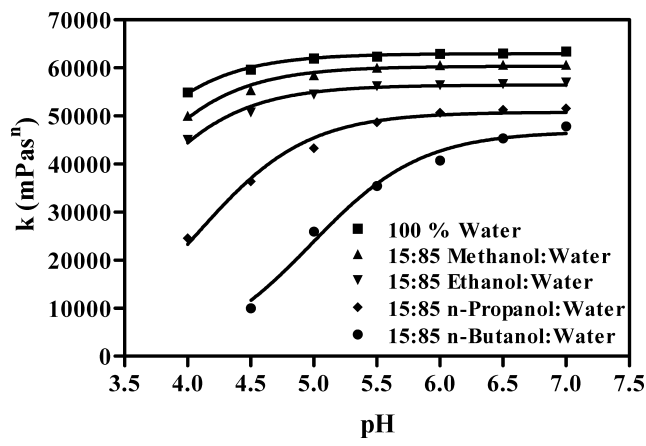

Fig. 1. Correlation between the Consistency Indexes $(k)$ of the Carbopol $^{\mathbb{R}}$ Ultrez $^{\mathrm{TM}} 10$ Hydroalcoholic Gels $(0.3 \% \mathrm{w} / \mathrm{w})$ and $\mathrm{pH}$ in the Different Alcohol Media (Theoretical Curves Obtained Using Eq. 1) the rate at which the system reached its maximum consistency $\left(k_{\max }\right)$. In general, this empirical model was able to adequately describe the variation of consistency indexes as a function of $\mathrm{pH}$ including variables with physical meaning.

The structure of Carbopol gels in solution is determined by the extent to which the macromolecules associate and form entanglement networks. In aqueous media, the polymer is mainly in the free acid form, and the $\mathrm{pH}$ induced generation of negative charges along the polymer chain, causes it to uncoil and expand. Subsequently, the value of the variable $\mathrm{pH}_{50}$ can be assessed as an inflection point in the structure forming effect of $\mathrm{pH}$, i.e. $\mathrm{pH}_{50}$ is the $\mathrm{pH}$ value required to generate half of the neutral gel's structure, expressed as $k_{\max } / 2$. From this $\mathrm{pH}$ value onward, the increase of consistency index with neutralisation is less pronounced, asymptotically reaching the maximum consistency $\left(k_{\max }\right)$ of the fully structured polymer network.

As can be deduced from the statistical parameters in Table 2 and the theoretical curves in Fig. 1, Eq. 1 adequately describes variation in the consistency index as a function of $\mathrm{pH}$ in the cosolvent: water mixtures studied. This finding extends considerably the range of practical application of the empirical models obtained on earlier occasions, ${ }^{2,22)}$ as well as their validity and applicability to the use of alcohol cosolvents with different degrees of polarity in the formulation of gelled systems for topical application.

The empirical variable, $k_{\max }$, obtained by application of the empirical model in Eq. 1, is thus directly related to the polarity and parameter of solubility of the alcohol utilised (Table 2 ). The $k_{\max }$ values decreased progressively in accordance with the composition of the jellifying medium: methanol, ethanol, $n$-propanol, $n$-butanol (Fig. 1 , Table 2). At the same time, an inverse relationship is observed in Table 2 between the variable $\mathrm{pH}_{50}$ and the solubility parameter. When the proportion of cosolvent: water $(15: 85 \% \mathrm{v} / \mathrm{v})$ is maintained, an decrease in the cosolvent polarity and solubility parameter tends to cause an increase in the $\mathrm{pH}_{50}$ value. This is, the degree of neutralization needed to obtain a half of the structural development. This increase is more pronounced between $n$ propanol and $n$-butanol than between methanol and ethanol (Table 2). ${ }^{31)}$

In addition, a certain degree of syneresis characterized by expulsion of interstitial liquid to the gel surface was observed in the case of the gels obtained from the $n$ butanol: water mixtures. The syneresis is due to the different polarity of $n$-butanol. On the other hand, this mechanism has also been associated with relaxation of the elastic stresses during gel setting and the resulting decrease in interstitial

Table 2. Correlation Parameters for the Mean Consistency Indexes $\left(\bar{k}, \mathrm{mPa} \cdot \mathrm{s}^{n}\right)$ of the Carbopol ${ }^{\circledR} \mathrm{Ultrez}^{\mathrm{TM}} 10 \mathrm{Gels}$ and $\mathrm{pH}$ (Eq. 1) in the Different Jellifying Media

\begin{tabular}{|c|c|c|c|c|c|c|}
\hline \multirow{2}{*}{$\begin{array}{l}\text { Cosolvent } \\
(\%, \mathrm{v} / \mathrm{v})\end{array}$} & \multirow{2}{*}{$\underset{\left(\mathrm{cal} / \mathrm{cm}^{3}\right)^{1 / 2}}{\delta_{\mathrm{T}}}$} & \multicolumn{5}{|c|}{$k=f(\mathrm{pH})$} \\
\hline & & $k_{\max }\left( \pm\right.$ S.E. $\left.^{a)}\right)$ & $\mathrm{pH}_{50}( \pm$ S.E. $)$ & $r$ & $\mathrm{~S}_{\mathrm{y}, \mathrm{x}}^{b)}$ & $\mathrm{ASS}^{c)}$ \\
\hline $100 \%$ Water & 23.4 & $62960 \pm 153.8$ & $3.180 \pm 0.022$ & 0.9896 & 340.0 & 57810 \\
\hline $15 \%$ Methanol & 14.5 & $60330 \pm 299.5$ & $3.340 \pm 0.034$ & 0.9774 & 657.1 & 215900 \\
\hline $15 \%$ Ethanol & 13.0 & $56430 \pm 327.6$ & $3.432 \pm 0.035$ & 0.9782 & 714.6 & 255300 \\
\hline $15 \% n$-Propanol & 12.0 & $50790 \pm 627.8$ & $4.072 \pm 0.038$ & 0.9870 & 1264.0 & 798600 \\
\hline $15 \% n$-Butanol & 11.3 & $46790 \pm 1175.0$ & $4.982 \pm 0.057$ & 0.9870 & 1808.0 & 1307000 \\
\hline
\end{tabular}


space available, which forces out the liquid. ${ }^{32)}$

The factors that influence hydrophilic polymeric gels viscosity are molecular size and shape, concentration, temperature, intermolecular forces of attraction of solvent and polymer and, of course, the degree of crosslinking of polyacrilic acid.

Other variables being constant, the above-described features can be related to changes in intermolecular forces of attraction, through variations in polymer hydration and solubility parameter induced by the alcohols. The ionised chains of Ultrez $^{\mathrm{TM}} 10$ polymer are extended in good solvents, as would be the case in aqueous media, due to hydrogen bond formation between water and hydroxyl groups of the gelling agent. In a poor solvent, the polymer chain would be more or less tightly coiled, preferring self-interactions to interaction with the solvent. The degree of hydration will hence determine the extent of formation of weak secondary bonds between polymer strands. Addition of alcohols may attract part of the water of hydration of the polymer, allowing the formation of more intermolecular secondary bonds, leading to precipitation. Furthermore, alcohols alter the solvent's characteristics, changing the solubility parameter; the $\mathrm{p} K_{\mathrm{a}}$-values of Carbopol $\left(\mathrm{p} K_{\mathrm{a}}=6\right)$ are changed due to the decreasing in the cosolvent polarity and solubility parameter, and so less dissociated product of Carbopol is formed, influencing also the hydration and viscosity of the polymer. By these mechanisms, the decreasing in the cosolvent polarity and solubility parameter decreases the maximum consistency, and increases the polymer neutralisation degree required to jelly the medium.

Addition of alcohols of decreasing polarity change the solvent's characteristics, reducing the total solubility parameter $\left[\delta_{\mathrm{T}} ;\left(\mathrm{cal} / \mathrm{cm}^{3}\right)^{1 / 2}\right]$ of the jellifying medium, as demonstrated by the direct second-order polynomial relationship obtained between $k_{\max }$ and $\delta_{\mathrm{T}}$ (Eq. 2, Fig. 2):

$$
\begin{aligned}
k_{\max }= & (-56760 \pm 11190)+(13010 \pm 1380) \cdot \delta_{\mathrm{T}}-(337.6 \pm 38.9) \cdot\left(\delta_{\mathrm{T}}\right)^{2} \\
& \left(r=0.9952 ; \mathrm{SAC}: 1690000 ; \mathrm{S}_{\mathrm{x}, \mathrm{y}}: 919.3\right)
\end{aligned}
$$

At the same time, the decrease in the solubility parameter of the mixture of solvents induces an increase in the empirical variable, $\mathrm{pH}_{50}$, i.e. to increase the degree of neutralisation required to obtain half structure development in the respective cosolvent:water mixture, as demonstrated by the inverse second-order polynomial relationship obtained between $\mathrm{pH}_{50}$ and $\delta_{\mathrm{T}}$ (Eq. 3, Fig. 2):

$$
\begin{aligned}
\mathrm{pH}_{50}= & (17.380 \pm 4.523)-(1.603 \pm 0.558) \cdot \delta_{\mathrm{T}}+(0.043 \pm 0.016) \cdot\left(\delta_{\mathrm{T}}\right)^{2} \\
& \left(r=0.9351 ; \mathrm{SAC}: 0.2762 ; \mathrm{S}_{\mathrm{x}, \mathrm{y}}: 0.3716\right)
\end{aligned}
$$

This findings point to the importance of the specific effect of the solvent on the conformational characteristics of the macromolecules, which determines the formation of the entanglement network, and the influence of this network on the viscosity of gels and empirical variables introduced in Eq. 1.

The Eqs. 2 and 3 demonstrate the relation between $k_{\max }$ and $\mathrm{pH}_{50}$ parameters with $\delta_{\mathrm{T}}$, respectively. That is, to see in what sense varies the rheological behaviour of gels and its degree of structuring with respect to the cosolvent solubility parameter. Verification of the validity of these expressions (Eqs. 2, 3) was made by graphically representing the $k_{\max }$ and $\mathrm{pH}_{50}$ values obtained as a function of $\delta_{\mathrm{T}}$. The regression coefficients of Eqs. 2 and 3 are all significant statistically at the 0.01 level. Consequently, equations has been considered to

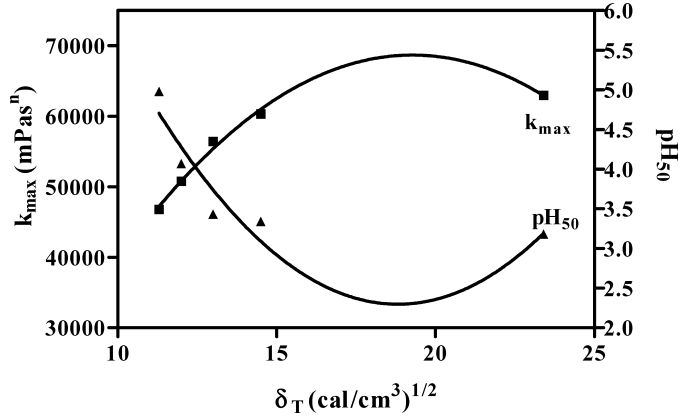

Fig. 2. Second Order Polynomial Correlations between the Empirical Variables $k_{\max }$ and $\mathrm{pH}_{50}$ of the Carbopol ${ }^{\circledR}$ Ultrez $^{\mathrm{TM}} 10 \mathrm{Gels}$ at $0.3 \% \mathrm{w} / \mathrm{w}$ and the Total Cosolvent Solubility Parameter $\left(\delta_{\mathrm{T}}\right)\left(\mathrm{cal} / \mathrm{cm}^{3}\right)^{1 / 2}$

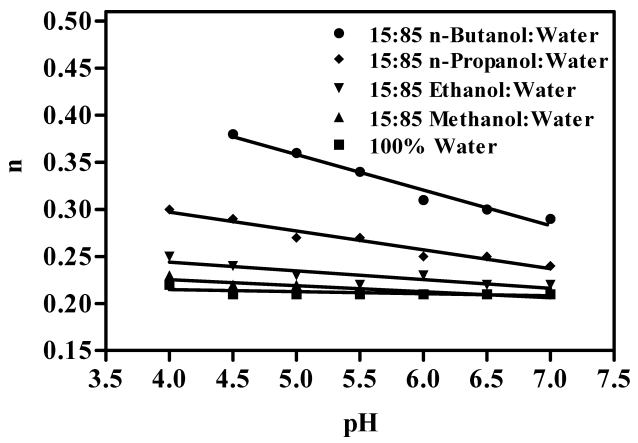

Fig. 3. Linear Correlation of the Flow Indexes $(n)$ of the Carbopol ${ }^{\circledR}$ Ultrez $^{\mathrm{TM}} 10$ Hydroalcoholic Gels $(0.3 \% \mathrm{w} / \mathrm{w})$ and $\mathrm{pH}$ in the Different Hydroalcoholic Mixtures

be sufficiently validated.

The maximum of $k_{\max }-\delta$ curve represents the maximum value of consistency index in the interval of cosolvent solubility studied, that would take place for a value around 19 $\left(\mathrm{cal} / \mathrm{cm}^{3}\right)^{1 / 2}$. The minimum of $\mathrm{pH}_{50}-\delta$ curve represents the minimum value of $\mathrm{pH}_{50}$ in the interval of cosolvent solubility studied, that would take place for a value around 19 $\left(\mathrm{cal} / \mathrm{cm}^{3}\right)^{1 / 2}$. Is the minimum value of $\mathrm{pH}_{50}$ required to generate half of the neutral gel's structure.

A cosolvent with a solubility parameter value of 19 $\left(\mathrm{cal} / \mathrm{cm}^{3}\right)^{1 / 2}$ would allow us to obtain both maxima consistency and degree of structure for Carbopol Ultrez 10 gels.

Flow Indexes Lastly, we examined the influence of $\mathrm{pH}$ on the power law flow indexes $(n)$ of the Carbopol ${ }^{\circledR}$ Ultrez $^{\mathrm{TM}}$ 10 gels in the different jellifying media to determine the respective empirical correlations with $\mathrm{pH}$ and cosolvent polarity. The pattern of correlation between the mean flow indexes $(\bar{n})$ and $\mathrm{pH}$ for the alcohol: water mixtures studied appears in graph form in Fig. 3. In all cases, the increase of $\mathrm{pH}$ was found to induce a nearly linear decrease of flow indexes, i.e. an increase in the pseudoplastic and shear thinning character of hydroalcoholic gels.

The statistical parameters of the equations of linear correlation between $n$ and $\mathrm{pH}$ are given in Table 3 . The slope of negative values obtained for each of the mixtures shows a non-Newtonian pseudoplastic behaviour, which goes up in relation to the increase in the final $\mathrm{pH}$ of the gel and in accordance with development of the final three-dimensional structure.

It is generally accepted that although the flow index $n$ measures departure from Newtonian flow, one of the best 
Table 3. Parameters of Linear Correlation between the Mean Flow Indexes $(\bar{n})$ of the Carbopol $^{\circledR}$ Ultrez $^{\mathrm{TM}} 10$ Gels and $\mathrm{pH}$ in the Different Jellifying Media

\begin{tabular}{|c|c|c|c|c|c|c|c|}
\hline \multirow{2}{*}{$\begin{array}{l}\text { Cosolvent } \\
(\%, \mathrm{v} / \mathrm{v})\end{array}$} & \multirow{2}{*}{$\begin{array}{c}\delta_{\mathrm{T}} \\
\left(\mathrm{cal} / \mathrm{cm}^{3}\right)^{1 / 2}\end{array}$} & \multicolumn{6}{|c|}{ Statistical parameters } \\
\hline & & $m^{a)}\left( \pm\right.$ S.E. $\left.{ }^{b)}\right)$ & $b^{c)}( \pm$ S.E. $)$ & $r$ & $\mathrm{~S}_{\mathrm{x}, \mathrm{y}}^{d)}$ & $F^{e)}$ & $P^{f)}$ \\
\hline $100 \%$ Water & 23.4 & $-0.0021 \pm 0.0012$ & $0.223 \pm 0.0069$ & 0.6124 & 0.0033 & 3.00 & 0.1438 \\
\hline $15 \%$ Methanol & 14.5 & $-0.0064 \pm 0.0015$ & $0.251 \pm 0.0086$ & 0.8825 & 0.0041 & 17.61 & 0.0085 \\
\hline $15 \%$ Ethanol & 13.0 & $-0.0093 \pm 0.0024$ & $0.281 \pm 0.0132$ & 0.8686 & 0.0063 & 15.36 & 0.0112 \\
\hline $15 \% n$-Propanol & 12.0 & $-0.0201 \pm 0.0020$ & $0.377 \pm 0.011$ & 0.9754 & 0.0053 & 98.00 & 0.0002 \\
\hline $15 \% n$-Butanol & 11.3 & $-0.0377 \pm 0.0032$ & $0.547 \pm 0.0185$ & 0.9861 & 0.0067 & 140.5 & 0.0003 \\
\hline
\end{tabular}

a) Slope, $b$ ) standard error, $c$ ) intercept, $d$ ) common error, $e$ ) $F$ of snedecor, $f$ ) probability.

way to view it is a function of the rate of change of structure with shear rate or shear stress. The structure can be altered on deformation by change in the shape of polymer molecules and in the number of molecular entanglements, possibly by straining a network of temporary junctions. As a result entanglement lifetimes determine changes in molecular shape and flow. On this basis, neutralisation will tend to decrease $n$ by increasing intermolecular forces of attraction, and hence increasing lifetime of junctions to produce a straining of the chain approaching that of a permanent network.

As mentioned above, the decrease of $n$ to a minimum value with the degree of neutralization shows the structuring effect of the $\mathrm{pH}$ factor, which generates a three-dimensional network that has a structure and properties similar to those found in a permanent network. ${ }^{33)}$ The flow index value, $n$, characteristic of the completely structured three-dimensional network at a neutral $\mathrm{pH}$ in the different jellifying media is determined by the intercept of the respective linear functions $n=f(\mathrm{pH})$ (Table 3).

One of the most important properties of the $n-\mathrm{pH}$ profiles obtained is the relationship between the intercept values and the total cosolvent solubility parameter $\left[\delta_{\mathrm{T}} ;\left(\mathrm{cal} / \mathrm{cm}^{3}\right)^{1 / 2}\right]$. The values calculated for intercepts of $n$ corresponding to each cosolvent: water mixture decrease progressively to a minimum value, which tends to remain constant, while the total solubility parameter increases (Table 3, Fig. 4).

As shown in Fig. 4, the existence of an asymptotic value as a bottom flow index for all the gels, demonstrates that the three-dimensional polymer lattice gradually becomes fully structured with increasing total solubility parameter.

This behaviour indicates a progressive accentuation of the pseudoplastic nature of the gels, that is, an increased departure from the ideal Newtonian behaviour as a function of the polarity of the jellifying medium. Considering the characteristics of this general correlation pattern, the intercept values were fitted by least-squares to an exponential decay function:

$$
n=\left(n_{\max }-n_{\min }\right) \cdot e^{-A \cdot \delta_{\mathrm{T}}}+n_{\text {min }}
$$

to obtain the following equation $\left[\delta_{\mathrm{T}}\right.$ : total solubility parameter, expressed in $\left.\left(\mathrm{cal} / \mathrm{cm}^{3}\right)^{1 / 2}\right]$ :

$$
\begin{aligned}
n= & 2033.8 \cdot e^{-0.776 \cdot \delta_{\mathrm{T}}}+0.203 \\
& \left(r=0.9825 ; \text { SAC: } 0.0012 ; \mathrm{S}_{\mathrm{x}, \mathrm{y}}: 0.0245\right)
\end{aligned}
$$

Equation 5 describes the exponential decrease of $n$ with the total solubility parameter and polarity of the cosolvent, a rate defined by empirical parameter, $A$, to a constant minimum value $\left(n_{\min }\right)$. The value of the factor $\left(n_{\max }-n_{\min }\right)$ determines the extent of dependence of $n$ and $\delta_{\mathrm{T}}$. This value $\left(n_{\max }-n_{\min }\right)$ is greater than those obtained in previous corre-

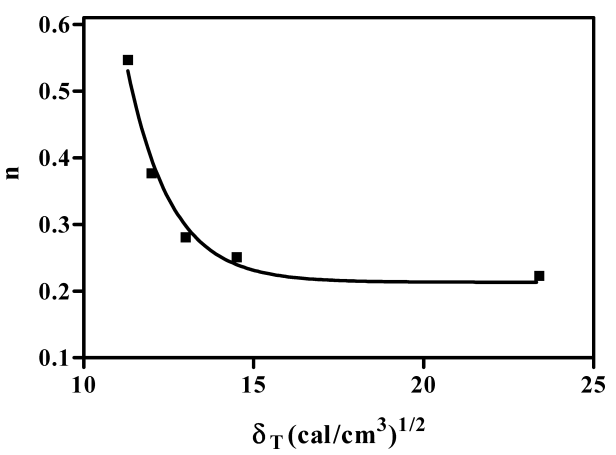

Fig. 4. Correlation between the Intercept Values of the Linear Functions $n=f(\mathrm{pH})$ and the Total Solubility Parameter $\left(\delta_{\mathrm{T}}\right)$

lations with polymer concentration, ${ }^{2)}$ meaning that the value of the flow index is highly sensitive to changes in the solubility parameter, especially in mixtures of less polarity. In these types of mixtures the incompatibilities increase between the hydrophilic polymer and the jellifying medium. The addition of alcohol attracts part of the polymer hydration water, enabling the formation of a larger proportion of secondary lattices, which alter the flow mechanism.

\section{Conclusion}

Globally, the results obtained indicate that from the rheological perspective, the Carbopol $^{\circledR}$ Ultrez $^{\text {TM }} 10$ hydroalcoholic gels behave as expected-i.e. as non-Newtonian pseudoplastic or shear thinning fluids.

The findings also suggest the interest of the polymer as a pharmaceutical excipient, for it can be used to obtain gels of adequate consistency as a function of the cosolvent and $\mathrm{pH}$. On the other hand, the working characteristics for obtaining a given consistency can be theoretically calculated from the proposed equations.

Gelation occurred at $\mathrm{pH} 5.5$ and is maintained to $\mathrm{pH} 7$. This is compatible with the physiological range of $\mathrm{pH}$ values and consequently, the polymer can be used as a hydrophilic excipient destined for cutaneous administration.

In general, this study of flow behaviour in methanol: water, ethanol:water, $n$-propanol: water and $n$-butanol: water gelled mixtures has demonstrated the existence of qualitatively similar functions to describe the dependence of consistency and flow indexes as a function of $\mathrm{pH}$. As a result, the influence of alcohol content and polarity on the structuring of the polymer network can be characterized using the empirical parameters obtained: maximum value of consistency index $\left(k_{\max }\right), \mathrm{pH}$ value needed to obtain $50 \%$ development of polymer network structure $\left(\mathrm{pH}_{50}\right)$ and asymptotic 
value of flow index $\left(n_{\min }\right)$.

Acknowledgements The authors would like to acknowledge financial support received from research project (Ref.: UAH PI2003/020), forwarded by the University of Alcalá, Madrid, SPAIN. The authors would like to thank Ms. M. F. Litzler for her skilled editorial assistance.

\section{References}

1) Arellano A., Santoyo S., Martin C., Ygartua P., Eur. J. Pharm. Sci., 7, 129-135 (1998).

2) Fresno M. J., Ramírez A., Jiménez M. M., Farmaco, 56, 437-441 (2001).

3) Vinogradov G. V., Malkin A. Y., "Rheology of Polymers," Chap. 1, 2, 3, 5, Springer-Verlag Berlin Heidelberg, New York, 1980.

4) Michailova V., Titeva S., Kotsilkova R., Krusteva E., Minkov E., Int. J. Pharm., 209, 45-56 (2000).

5) Pharmaceutical Press and the American Pharmaceutical Association, "Handbook of Pharmaceutical Excipients," ed. by Rowe R. C., Sheskey P. J., Weller P. J., Pharmaceutical Press and the American Pharmaceutical Association, Washington, 2003.

6) "Carbopol Resins Handbook, Specialty Chemicals," BFGoodrich Company, 9921 Brecksville Road, Cleveland, Ohio 44141-3247, 1997.

7) "Polymers for Pharmaceutical Applications, I. General Overview (Technical Reference)," BFGoodrich Company, 1997.

8) Tamburic S., Craig D. Q. M., J. Controlled Release, 37, 59-68 (1995).

9) Shawesh A. M., Kaukonen A., Kallioinen S., Antikainen O., Yliruusi J., Pharmazie, 58, 130-135 (2003).

10) Barry B. W., Meyer M. C., Int. J. Pharm., 2, 1-25 (1979).

11) Picout D. R., Ross-Murphy S. B., Scientific WorldJournal, 3, 105 121 (2003).

12) Craig D. Q. M., Tamburic S., Buckton G., Newton J. M., J. Controlled Release, 30, 213-223 (1994).

13) Rodríguez R., Alvárez-Lorenzo C., Concheiro A., Biomacromolecules, 2, 886-893 (2001).

14) "Carbopol ${ }^{\circledR}$ Ultrez $^{\mathrm{TM}}$, The Polymer as Universal as Water, Specialty Chemicals," BFGoodrich Company, 9921 Brecksville Road, Cleveland, Ohio 44141-3247, 1997.

15) Desai D. D., Hasman D. F., Schmucker-Castner J. F., "Advances in Car- bomer Polymer Technology," BFGoodrich, Technical data sheets for Carbopol ${ }^{\mathbb{R}}$ Ultrez 10 Polymer, Specialty Chemicals, 1998.

16) Ho H. O., Huang F. C., Sokoloski T. D., Sheu M. T., J. Pharm. Pharmacol., 46, 636-642 (1994).

17) Hosoya O., Sano M., Wada Y., Seki T., Sugibayashi K., Juni K., Morimoto Y., Chem. Pharm. Bull., 46, 882-885 (1998).

18) Raghavan S. L., Trividic A., Davis A. F., Hadgraft J., Int. J. Pharm., 193, 231-237 (2000).

19) Edsman K., Carlfors J., Petersson R., Eur. J. Pharm. Sci., 6, 105-112 (1998).

20) Ferrari F., Rossi S., Bonferoni M. C., Caramella C., Boll. Chim. Farm. 140, 329-336 (2001).

21) Rossi S., Ferrari F., Bonferoni M. C., Caramella C., Boll. Chim. Farm., 140, 337-344 (2001).

22) Ramírez A., Fresno M. J., Jiménez M. M., Selles E., Pharmazie, 54, 444-447 (1999).

23) Herráez J. V., Dolz M., Sobrino P., Belda R., González F., Pharmazie, 48, 359-362 (1993).

24) Dolz M., Herráez M., González F., Díez O., Delegido J., Hernández M. J., Pharmazie, 53, 126-130 (1998).

25) Bentley M. V., Marchetti J. M., Ricardo N., Ali-Abi Z., Collett J. H., Int. J. Pharm., 193, 49-55 (1999).

26) Sanz T., Martin-Villodre A., Pla-Delfina J. M., Herráez J. V., Int. J. Pharm., 233, 43-50 (2002).

27) Nakanishi T., Kaiho F., Hayashi M., Chem. Pharm. Bull., 46, 171173 (1998).

28) Amsellem E., Derrien F., Lanquetin M., Paris J., Marty J. P., Arzneim.Forsch., 48, 492-496 (1998)

29) Agarwal V., Singh S. K., Reddy I. K., Durrani M. J., Khan M. A. Drug Dev. Ind. Pharm., 25, 659-665 (1999).

30) Martin A., Bustamante P., An. R. Acad. Farm., 55, 175-202 (1989).

31) Chu J. S., Yu D. M., Amidon G. L., Weiner N. D., Goldberg A. H., Pharm. Res., 9, 1659-1663 (1992).

32) Zatz J. L., Kushla G. P., "Pharmaceutical Dosage Forms: Disperse Systems," ed. by Lieberman H. A., Rieger M. M., Banker G. S., Marcel Dekker Inc., New York, 1989, pp. 495-510.

33) Norton I. T., Jarvis D. A., Foster T. J., Int. J. Biol. Macromol, 26 255-261 (1999). 